\title{
A Methodology for Logistics Partners' Selection to Compose Virtual Organizations Based on KPI
}

\author{
Omir Correia Alves Junior ${ }^{1}$ and Ricardo J. Rabelo ${ }^{2}$ \\ ${ }^{1}$ Dept. of Computer Science, \\ State University of Santa Catarina, Brazil \\ omalves@gmail.com \\ ${ }^{2}$ Dept. of Automation and Systems Eng., \\ Federal University of Santa Catarina, Brazil \\ rabelo@das.ufsc.br
}

\begin{abstract}
This paper presents an exploratory and qualitative work of a novel model for the selection of the most adequate logistics providers that will compose a virtual organization. It includes a performance measurement model and a supporting methodology that considers the intrinsic dynamics, autonomy and temporality of Virtual Organizations, involving both intra and interorganizational indicators at strategic levels. The model is flexible in terms of both allowing performance indicators' weights relaxation and being adapted according to the organizations' governance model.
\end{abstract}

Keywords: virtual organization, logistics partners' selection, performance indicators, AHP method, performance measurement.

\section{Introduction}

The Globalization is a trend that favors the expansion of logistics partners. New markets and new products have been increasingly created all over the world and proper logistic providers should be hired. The cleverer this activity is done the greater visibility, improved customer service, better planning and cost savings will be supported [1]. When companies work in more volatile strategic networks, the difficult of selecting the most appropriate logistic providers is even higher. This works focuses on the Virtual Organization (VO) type of network.

Part of such difficulty is due to the intrinsic nature of a VO, which is a temporary and dynamic strategic alliance of autonomous, heterogeneous and usually geographically dispersed companies (often SMEs) created to attend to very particular business opportunities [2-4], sharing costs, benefits and risks, acting as it was one single enterprise [5, 6]. After ending all legal obligations a VO is dismissed.

Differently from traditional supply chains, logistics providers (responsible for handling material and cargo transportation) are not known in advance, as this depends on the business, on the client, on the country or region's regulations, etc. Therefore, the collaboration among involved industrial partners (who produces some part of the good) and logistic providers are crucial to be augmented [7, 8] as a way to fulfill temporal and quality requirements of this unique business opportunity as well as to 
differentiate in the market as long as they create value in this chain $[9,10]$. Considering this tighter collaboration, logistics providers are seem as logistics partners (LP) and a business opportunity is as a collaboration opportunity (CO).

Most of the works found out in the literature tackled this problem usually calling it as partner's search and selection, but with the focus on the selection of industrial partners (IP) instead of on logistics partners. Besides that, the works on logistics in VOs essentially considers performance indicators basically at operational level, neglecting the also important strategic level. Yet, they do not consider other relevant dimensions when autonomous and collaborative companies do business together, such as governance and trust. Finally, the large majority of the works try to apply an automatic approach when selecting partners. We argue that the selection of LPs in a VO scenario is so complex and full of particularities that making this automatically is perhaps unrealistic as well as it prevents involved companies' managers from properly putting in practice their experience.

This work proposes a novel model that complements other contributions about IPs' selection, supporting the suggestion of adequate LPs for given VO.

This paper is organized as following: section I introduced the problem; section 2 details the problem and contextualizes it within the collaborative networks area; section 3 presents the supporting methodology for LP suggestion; section 4 provides an example of the model usage; and section $\mathrm{V}$ provides some preliminary conclusions about the model.

\section{Logistic Network Problem}

A VO is one type of a diversity of Collaborative Network Organizations (CNO). However, a key aspect when considering VOs is that all of its members come from another type of $\mathrm{CNO}$, which is the so-called Virtual organization Breeding Environment (VBE). A VBE is a long-term alliance of companies whose ultimate goal is to be the basis for the creation of VOs. Likely VOs, VBE members are also composed of autonomous, heterogeneous and geographically dispersed companies. Regarding that they share principles and working methods, the creation of VOs from a VBE becomes much faster, more effective and less complex to manage [11, 12]. A typical VO is generally composed of LPs and IPs, creating a logistics and value chain network, as illustrated in Figure 1.

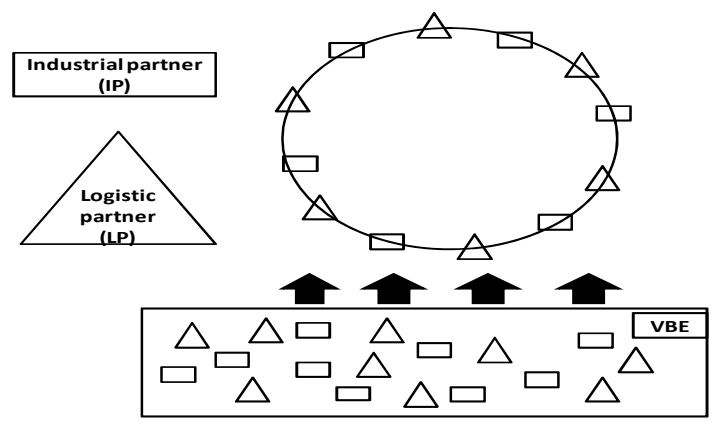

Fig. 1. VO composed of LPs and IPs 
The reference process for a VO creation consists of seven steps (Figure 2) [13]. Adopting a performance measurement approach, in [14] partners' search and selection step is extended introducing key performance indicators (KPI) as a first task to be made to filter IPs. This paper follows the same approach, adapting it to LPs and restricting the model to the suggestion stage. This is a complex task because LPs will work collaboratively in a $\mathrm{VO}$ and their selection should consider particular aspects of a VO and VBE, such as:

- $\quad$ LPs can only be identified after knowing the particular CO in details;

- A repeated CO will be rarely composed of the same set of VBE members;

- VO's LPs and IPs not necessarily will have worked together in previous COs;

- COs are usually unique or one-of-a-kind;

- $\quad \mathrm{KPI}$ and/or their weights vary from one CO to another;

- LPs usually have different information system, semantics and performance measures;

- $\quad$ The final handshake among IPs and LPs should be carried out as fast as possible;

- $\quad$ Each VBE has its particular governance model.

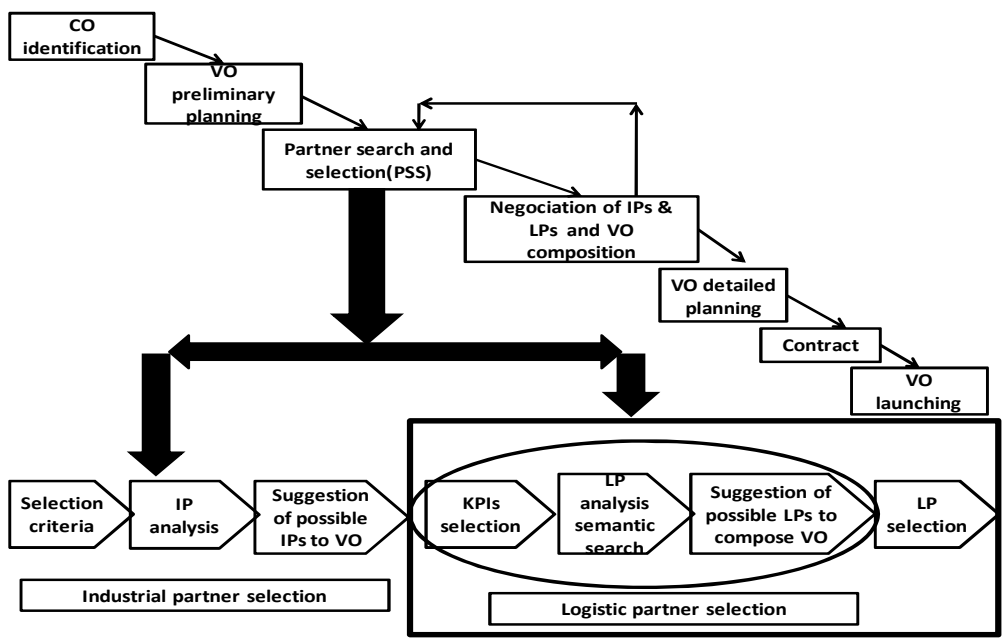

Fig. 2. Extended framework for the VO creation

A literature survey about this showed that there are several approaches based on performance measurement and KPIs for evaluating companies [15]. However, few of them address the problem of KPI models for LPs in VOs. For example, in [16] a generic methodology based on balanced Scorecard (BSC) indicators is proposed, but without any support for governance and trust issues. The methodology proposed by [17] assigns performance indicators for each partner but does not provide criteria for analyzing the collaboration level of partners to compose VOs. In [18] the complexity of structuring a methodology for VO partners' selection is identified and a hierarchical methodology based on multiple attribute decision making is proposed. However this work has focused only on intra-organizational performance indicators. 


\section{Proposed Methodology}

In order to tackle this issue of suggesting adequate LPs for a given VO a methodology is proposed (Figure 3). Two important assumptions are considered. Firstly, all LPs are members of a VBE. Secondly, there is a global coordinator of the process, which is called VO Coordinator. An additional role is taken by a logistic specialist, who permanently audits the LPs' KPI values.

\section{A. CO Identification}

In this first methodology stage the $\mathrm{CO}$ is verified in order to identify the logistics itineraries that have to be carried out. A CO, besides other information, is composed of logistics-related data showed on section 4 . This set of information was based on a VO information reference model [19] [14] and extended for this work.

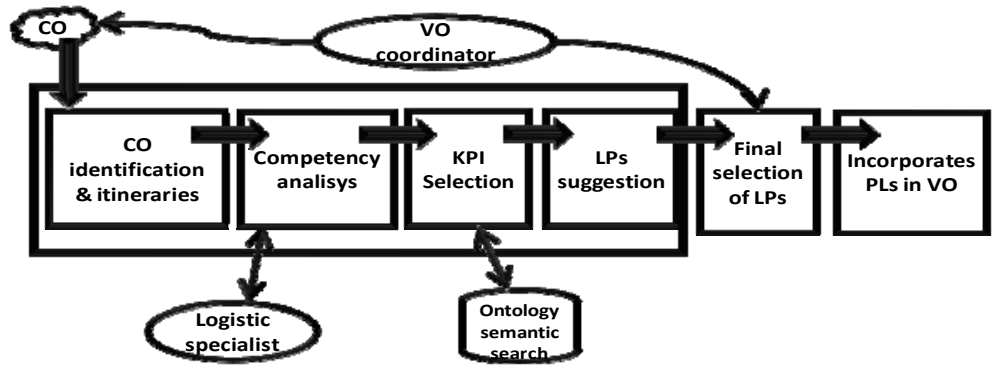

Fig. 3. LP selection steps

\section{B. LP competency skills analysis}

In a first round of analysis the methodology checks the technical LP's competences against to every single CO itinerary. If a given LP is pre-selected then it is moved to a suggested list for further VO coordinator decision. After an analysis on the logistics discipline [20], seven attributes were elicited to represent LP competences showed on section 4. The formal competency skills analysis is performed using the set theory. Two sets are considered: $R$ and $M . R$ represents the whole set of specific CO requirements $(R=\{1, \ldots, r\})$. $M$ represents the set of LP's competencies $(M=\{1, \ldots, m\})$. The problem is to find a match between $R$ and $M$, which will then define the preselected PLs for the given CO. This is provided by the function $G(i, j)$, which represents the intersection of $R$ and $M$ sets:

$$
G(i, j)=|R i \cap M j|, \forall i \in R \wedge \forall j \in M
$$

Where:

$i=$ number of PLs; $j=$ number of COs.

\section{Identification and selection of KPIs}

The third step of the methodology aims at selecting the most appropriate KPIs that will be applied over every CO's itinerary to filter the pre-selected LPs. This process is aided by an ontology, which links the semantic of CO's attributes with the KPI model (Figure 3). It is used to provide a formal representation that can be used and reused to 
facilitate understanding of all involved concepts and relationships between them in a specific domain [21]. The developed KPI model comprises fifteen KPIs (see section D). More than one KPI can be applied to evaluate a given CO itinerary. LPs will be selected after this set of KPI evaluation. This strategy has been inspired from [14]. With the selected list of KPIs and LPs, the methodology determines the level of collaboration (LC) (see section G) of each pre-selected LP for each itinerary. The LP to be finally suggested is the one with the highest LC value or with the highest coefficient of regression. VO coordinators evaluate the suggested list and assign one LP to the given itinerary and then this LP becomes member of the VO. The method repeats until the end of the $\mathrm{CO}$ itineraries, i.e. when there is one or a set of LPs selected to link all the involved (and previously defined) VO's industrial partners.

\section{KPI model}

A crucial element in the methodology is the KPI model. Regarding this, two general requirements were necessary to cope with. Firstly, the set of KPIs should consider both intra and inter organizational perspectives. Secondly, they should also consider indicators at strategic level. After a literature review, several KPI models were found out (e.g. [22-25]). However, none of them were neither comprehensive enough to cope with those requirements nor were devoted to logistics in dynamic alliances (e.g. VOs). The devised KPI model has considered some existing models (e.g. SCOR [26]) and complemented with a literature overview. It is composed of fifteen KPIs:

- $R O E$ (return-on-equity): The amount of net income returned as a percentage of shareholders equity;

- Cash flow: focusing on the cash being generated related to how much is being generated and the safety net it provides to the LP;

- Cost Control: controls the cost reduction of LPs;

- Customer satisfaction: measures the customer perception related to delivered services;

- Susceptibility: the elapsed time between customer purchase order and product(s) delivery;

- Commitment: measures the level of commitment between the LPs;

- Collaboration: measures the LPs level of collaboration;

- IT maturity: measures if the LP's IT objectives are aligned to its business strategies;

- Governance: measures how is the code of conduct and cultural issues of each LPs;

- Flexibility: measures the LP flexibility to adapt to changes along VO operation;

- Environmental performance: measures how the LP copes with environmental practices;

- Availability: measures the level of LP availability;

- Effectiveness: measures if resources (e.g. labor) are properly allocated;

- Trust: measures the level of trust between the LPs;

- Communication: measure the level of effective communication among LPs' members.

Each KPI is seen as a strategic dimension, which is divided into a subset of individual and operational/lower level performance indicators (PIs). When computed as a whole, they provide the value of the KPI itself. For example, KPI Cost Control is calculated considering the PIs cost of warehousing, reverse cost and labor cost.

\section{E. Assigning weights to KPIs}

The methodology applies the AHP method to assign weights to the fifteen KPIs. AHP was proposed in [27] to solve multiple criteria problems in a hierarchical structure. In 
AHP, criteria related to the goal are distributed at lower levels from the top of the KPI weight structure (Figure 4).

The LC calculation (see section G) uses this hierarchical structure to distribute weights (i.e. their importance) of KPIs and hence to suggest the most suitable LPs. All KPIs are weighted. By default, the methodology assigns the higher weights to KPIs with makes a semantics matching with the $\mathrm{CO}$, whereas lower weights are assigned to those without matching. The VO coordinator is in charge of assignment weights to KPIs. If necessary, weights can be redefined along the process.

\section{F. Assign values to KPIs}

As the model works also based on historical data, the VBE database should be updated with the applied KPIs values after the VO dissolution. This is done by all the involved VO's companies via electronic questionnaires. Likert scale [28] is used to normalize KPI values, defining values from zero to five. These values are calculated from the set of tactical performance indicators that composes each (strategic) KPI.

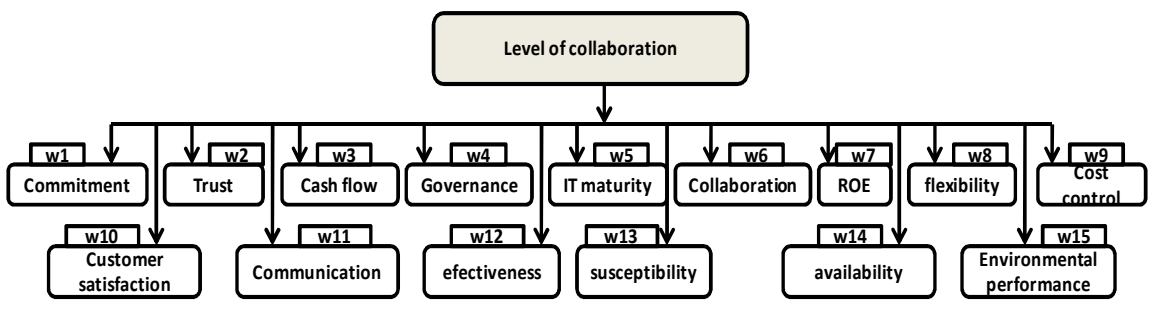

Fig. 4. Using AHP with the KPIs

\section{G. Level of collaboration (LC)}

The final decision about which LPs will compose a VO is determined by a last filter, which is LC. LC is a value calculated for each LP that was selected by the competence analysis. It is represented by a vector of collaboration (VC), which is formed by the historical collaboration of each pre-selected LP in past VOs (Figure 5).

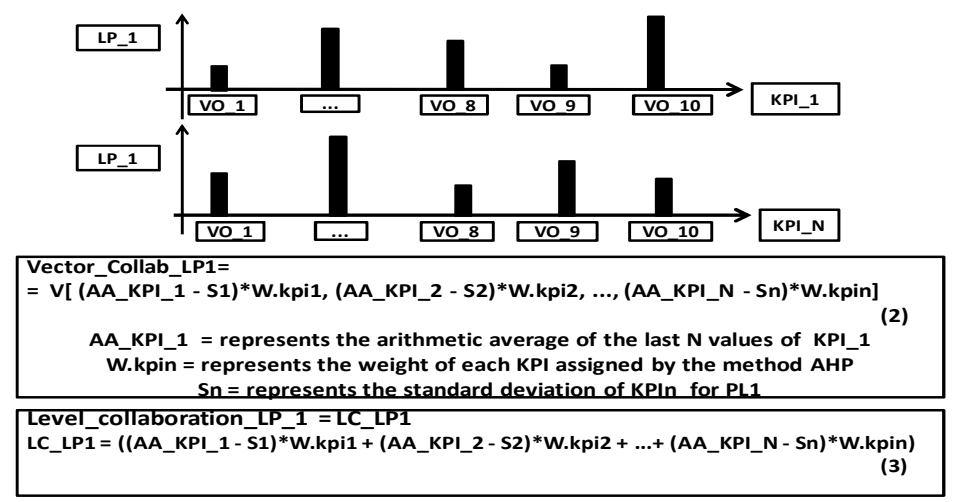

Fig. 5. Value of KPIs and level of collaboration formula 
LC is composed of a set of "positions" (KPIs), where each position is calculated multiplying the average of the historical values of each KPI minus the standard deviation for each KPI by its respective weight. The determination of the LC is applied to all LPs, as follows:

- Get past KPI values (from the VBE database) from each pre-selected LP associated to their previous VOs;

- Determine the time horizon to be applied upon the LPs. Depending on the VO or the VBE policy, this can vary from a number of past VOs (e.g. the last ten participations) or period of time (e.g. the participation in the last two years);

- Calculates Vector of Collaboration (VC), where each vector field is the arithmetic average of the last values minus the standard deviation multiplied by the respective KPI's weight for each KPI. The weight is represented by the variable W.idn, and it is calculated using the AHP method;

- Determine the total LC for each LP by the sum of the indices of the respective KPI VCs;

- Determine the straight line for the range of last $n L C$ values for each LP and its coefficient, using the regression theory and the minimum quadratic method. (Figure 6);

- Suggests the LP for each CO's itinerary according to the highest LC and/or the positive coefficient of regression of the straight line. The positive coefficient means that, historically, after each VO the KPIs values of a LP are increasing.

The formula for $\mathrm{VC}$ and $\mathrm{LC}$ calculation is given by:

$$
V C(i, j, k)=\left(A A \_K P I(i, j, k)-S(i, j, k)\right) * W(i, j, k)
$$

where:

$i=$ amount of KPIs; $j=$ number of LPs per itinerary; $k=$ number of itineraries within a CO;

$A A \_K P I=$ arithmetic average of historical values of the KPI $i$, referring to PL $j$, which is associated with the itinerary $k$;

$W(i, j, k)=$ weight assigned to KPI $i$ by AHP;

$S(i, j, k)=$ standard deviation of $K P I i, L P j$, and itinerary $k$;

$$
L C(j, i s)=\sum_{j=1}^{1 B} V C(i, j, i s)
$$

where:

$i=$ number of KPIs; $j=$ number of LPs by itinerary; $k=$ number of itineraries of the CO;

$V C(i, j, k)=$ Vector of collaboration from KPI $i$ to partner $j$, related to itinerary $k$;

$L C(j, k)=$ level of collaboration of the PL $j$ to itinerary $k$;

$L C \_a(j, k)=$ represents the $L C$ of the $P L j$ to itinerary $k$ considering its coefficient of regression value;

$$
L C(k)=\left[\operatorname{Max}(L C(j, k)) \text { or Max }\left(L C \_a(j, k)\right)\right]
$$

where:

$L C(k)$ :represents the greatest value for the $L C$ or for the $L C$ with the highest coefficient of regression to itinerary $k$. 


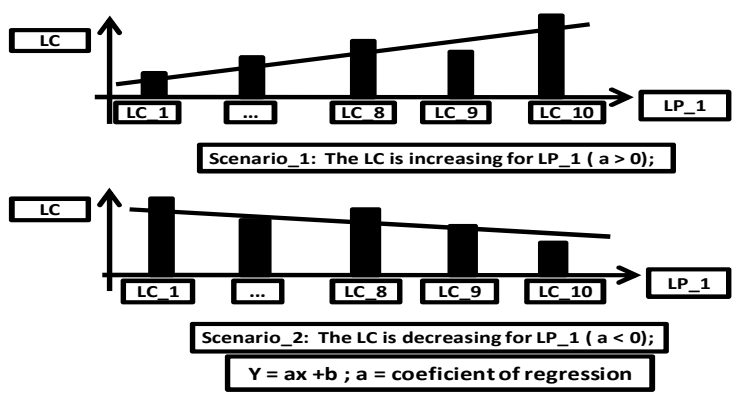

Fig. 6. Linear regression analysis for LC

\section{Example of the Methodology Usage}

Figure 7 generally illustrates an example on how the proposed model can be used. The given $\mathrm{CO}$ refers to a transportation of some good from the location $\mathrm{A}$ to $\mathrm{B}$, with three legs in between: A-C, C-D and D-B. Each leg is represented by a CO's itinerary and they require up to three LPs to deliver the involved parts to the industrial partners. A LP may even get all the three itineraries, depending on its LC. CO's details are:

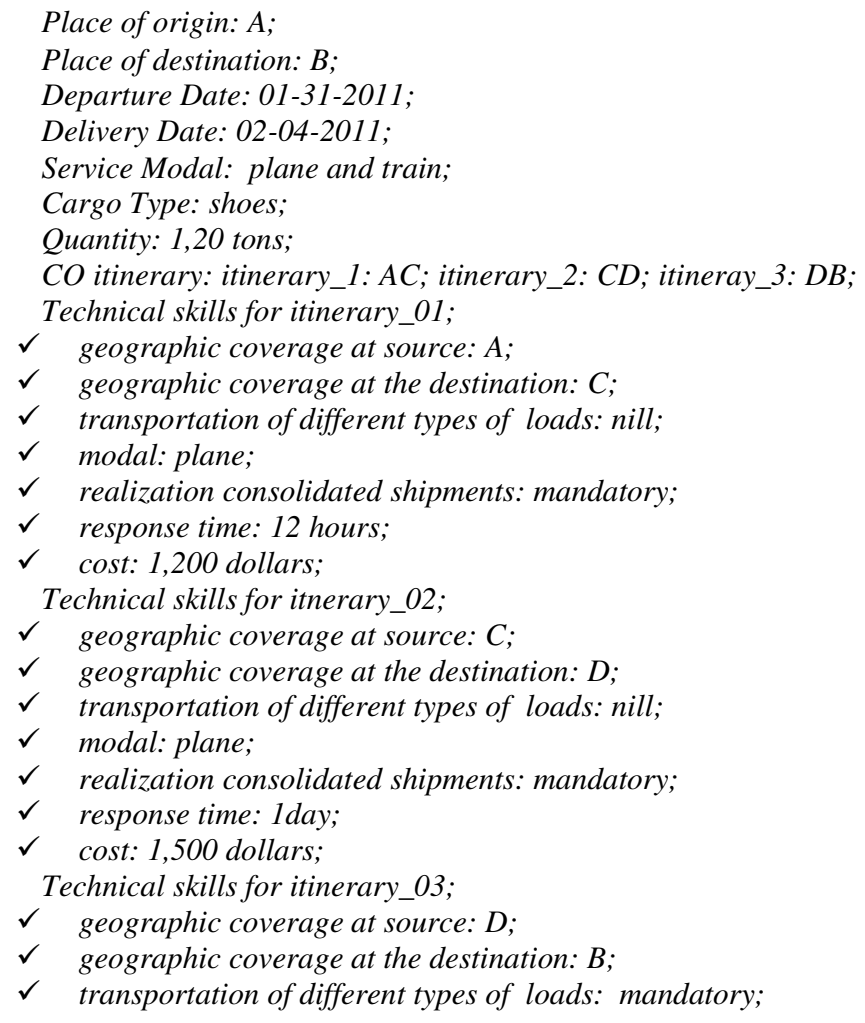


$\checkmark$ modal: train;

$\checkmark$ realization consolidated shipments: mandatory;

$\checkmark$ response time: 1 day;

$\checkmark$ cost: 1,800 dollars;

- $\quad$ Level of Collaboration (LC): 5,1.

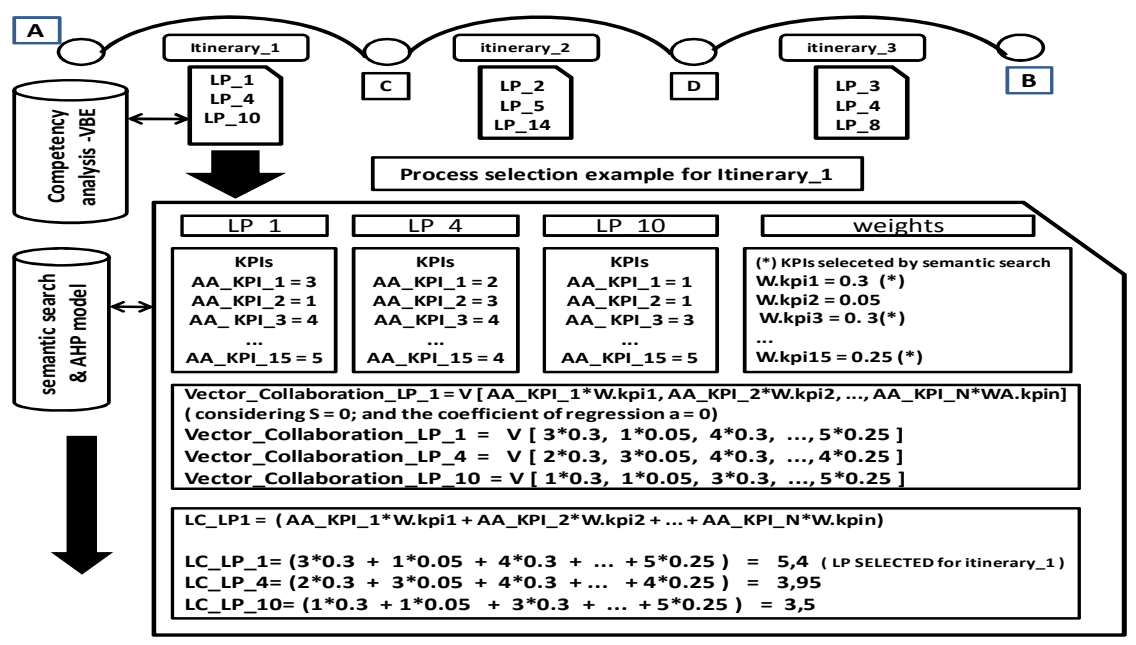

Fig. 7. Example of LP selection

As explained in Section 3, the selection of the set of LPs to be suggested to VO coordinator to compose $\mathrm{VO}$ related to the given $\mathrm{CO}$ is carried out along five phases:

- Phase 1: the actor who gets the CO (e.g. the VO coordinator, a VBE broker or the industrial partner who was directly contacted by the customer, depending on the VBE operating rules checks the itineraries associated with the $\mathrm{CO}$;

- $\quad$ Phase 2: for each CO's itinerary the VBE database is consulted and LPs are preselected based on their competencies. In the example, $L P \_1, L P \_4$ and $L P \_10$ would be pre-selected for itinerary_1;

- Phase 3: Semantic search selects KPIs which are related to the OC, and weights are assigned to them (using AHP method);

- Phase 4: calculation of: i) the arithmetic average for each KPI; ii) the standard deviation for each KPI (in this example, $S n=0$, and so $a=0$ ); iii.) the VC for each LP; iv) the final LC for all LPs previously selected;

- $\quad$ Phase 5: For each itinerary the LP with the highest LC is suggested to compose the VO. For example, $L P \_l$ would be selected for itinerary_l with the score 5.4 ;

The process repeats for all OC's itineraries ( 2 and 3 in this case). In the case of problems during the VO execution (or before its effective starting) that cause the need for a LP replacement, the second (and so forth) LP of the list is contacted to see if it is still available. If none of them is available or the VO coordinator considers that they are no longer useful, the methodology is started again from the competence analysis 
phase on. In the worst case, if none LP is found out, the client should be contacted to see at which extent some relaxations (e.g. in the delivery time) are allowed. Otherwise the VO should be cancelled.

\section{Conclusions}

This paper has presented a novel model and supporting methodology for the selection of the most adequate logistics partners to compose a Virtual Organization (VO). It corresponds to an ongoing and essentially exploratory and qualitative work. The complexity of the problem refers to the intrinsic dynamics, temporality and autonomy of VO, whose partners (including logistics ones) can only be identified when the business opportunity is got. Therefore, it is of extreme importance to not only make this process faster but also with more quality and confidence.

A new performance model has been presented and it is devoted to cope with the singularities of VO. It is composed of 15 KPIs and it is used to compare companies' competences, which can be flexibly assigned via AHP method. A formal ontology to establish the relation among requirements, KPIs and competences was specially conceived so mitigating semantic problems. The set of logistics partners are selected based on their level of collaboration, also considering their past performance.

So far the model has been verified in a controlled environment and close to a small group of logistics operators in the form of general questionnaires. Next main steps include the development of a decision support system prototype that implements the whole model's environment, and testing it in near real scenarios.

\section{References}

[1] Mentzer, J.T., Konrad, B.P.: An efficiency/effectiveness approach to logistics performance analysis. J. of Business Logistics 12(1), 33-62 (1991)

[2] Camarinha-Matos, L.M., Afsarmanesh, H.: Collaborative Networks: A New Scientific Discipline. J. of Intelligent Manufacturing 16(4), 439-452 (2005)

[3] Mowshowitz, A.: Virtual organization. Com. of the ACM 40(9), 30-37 (1997)

[4] Katzy, B.R., Obozinski, V.: Designing the virtual enterprise. In: Proceeding of ICE 1999, 5th Int. Conf. On Concurrent Enterprising, pp. 11-20 (1999)

[5] Sandberg, E.: Logistics collaboration in supply chains: practice vs. theory. The Int. J. of Logistics Management 18(2), 274-293 (2007)

[6] Camarinha-Matos, L., Afsarmanesh, H.: Collaborative Networks: Reference Modeling. Springer, New York (2008)

[7] Skjoett-Larsen, T.T., Andresen, C.: Supply chain collaboration Theoretical perspectives and empirical evidence. Int. J. of Physical Distribution and Logistics Management 33(6), 531-549 (2003)

[8] Whipple, J.M., Russell, D.: Building supply chain collaboration: a typology of collaborative approaches. Int. J. of Logistics Management 18(2), 174-196 (2007)

[9] Rafele, C.: Logistic service measurement: a reference framework. J. of Manufacturing Technology Management 15(3), 280-290 (2004) 
[10] Esper, T.L., Williams, L.R.: The value of collaborative transportation management (CTM): its relationship to CPFR and information technology. Transportation Journal 42(4), 55-65 (2003)

[11] Rabelo, R.J., et al.: Smart Configuration of Dinamic Virtual Enterprises. In: Fifth IFIP Working Conference on Virtual Enterprises, pp. 193-204 (2004)

[12] Afsarmanesh, H., Camarinha-Matos, L.M.: A Framework for Management of Virtual Organization Breeding Environments. In: Sixth IFIP Working Conference on Virtual Enterprises, pp. 35-48 (2005)

[13] Camarinha-Matos, L.M., et al.: Towards a Framework for Creation of Dynamic Virtual Organizations. In: 6th IFIP Work. Conf. on Virtual Enterprises, pp. 69-80 (2005)

[14] Baldo, F., Rabelo, R., Vallejos, R.V.: Modeling Performance Indicators' Selection Process for VO partners' Suggestion. In: 8th IFIP International Conference on Information Technology for Balanced Automation Systems, pp. 67-76 (2008)

[15] Seifert, M.: Collaboration formation in Virtual Organisations by applying prospective performance measurement. Phd Thesis, University of Bremen (2009)

[16] Westphal, I., Thoben, K., Seifert, M.: Measuring collaboration performance in virtual organizations. In: 8th IFIP Working Conf. on Virtual Enterprises, pp. 33-42 (2007)

[17] Bititci, U.S., et al.: Measuring and managing performance in extended enterprises. Int. J. of Operations \& Production Management 25(4), 333-353 (2005)

[18] Sarkis, J., Talluri, S., Gunasekaran, A.: A strategic model for agile virtual enterprise partner selection. Int. J. of Operations \& Production Manag. 27(11), 1213-1234 (2007)

[19] Camarinha-Matos, L.M., Afsarmanesh, H.: Elements of a base VE infrastructure. Computers in Industry 51(2), 139-163 (2003)

[20] Gunasekaran, A., Patel, C., Tirtiroglu, E.: Performance measures and metrics in a supply chain environment. Int. J. of Operations and Production Management 21(1/2), 71-87 (2001)

[21] Berners-Lee, T., Hendler, J., Lassila, O.: The semantic Web. Scientific American 284(5), 28-37 (2001)

[22] Saiz, J.J.A., Bas, A.O., Rodríguez, R.R.: Performance measurement system for enterprise networks. Int. J. of Productivity and Performance Management 56(4), 305-334 (2007)

[23] Westphal, I., Thoben, K.D., Seifert, M.: Measuring Collaboration Performance in Virtual Organizations. In: 8th IFIP Work. Conf. on Virtual Enterprises, pp. 33-42 (2007)

[24] Kim, D.H., Kim, C.: A generic framework of performance measurement in networked enterprises. In: Camarinha-Matos, L.M., Paraskakis, I., Afsarmanesh, H. (eds.) PRO-VE 2009. IFIP AICT, vol. 307, pp. 259-265. Springer, Heidelberg (2009)

[25] Seifert, M., Wiesner, K., Do, T.: Prospective performance measurement in virtual organizations, pp. 319-326 (2008)

[26] Supply-Chain Council, Supply Chain Operations Reference Model (2006)

[27] Saaty, T.L.: How to make a decision: the analytic hierarchy process. European Journal of Operational Research 48(1), 9-26 (1990)

[28] Linacre, J.M.: Optimizing rating scale category effectiveness. J. Appl. Meas. 3(1), 85-106 (2002) 